\title{
Thermal conductivity of PDMS filled with hollow glass microspheres
}

\author{
Sergei Vlassov ${ }^{1,2^{*}}$, Sven Oras ${ }^{2,3}$, Martin Timusk ${ }^{2,4}$, Vahur Zadinn ${ }^{5}$, lya M. Sosnin ${ }^{1}$, Rünno Lõhmus ${ }^{2}$, Leonid M. \\ Dorogin $^{1}$ \\ ${ }^{1}$ ITMO University, Kronverskiy pr., 49, 197101 Saint-Petersburg, Russia \\ ${ }^{2}$ Institute of Physics, University of Tartu, W. Ostwaldi Str. 1, 50412, Tartu, Estonia \\ ${ }^{3}$ Tallinn University of Technology, Tartu College, Puiestee 78, Tartu, 51008, Estonia \\ ${ }^{4}$ Research Laboratory of Functional Materials Technologies, Faculty of Materials Science and Applied \\ Chemistry, Riga Technical University, Paula Valdena 3/7, 1048 Riga, Latvia \\ ${ }^{5}$ Institute of Technology, University of Tartu, Nooruse 1, 50411, Tartu, Estonia
}

*Corresponding author. Tel.: +37255941841

E-mail address: sergei.vlassov@ut.ee (S. Vlassov)

\begin{abstract}
Polydimethylsiloxane (PDMS) is the most widely used silicon-based polymer due to its versatility and the range of attractive properties. Fabrication of PDMS involves liquid phase cross-linking to obtain hydrophobic and mechanically flexible material in the final solid form. This gives opportunity to add various fillers to affect the properties of resulting material. In the present work, we describe simple and reliable method of making a PDMS-based composite material with significantly improved thermal insulation properties by adding hollow glass microspheres (HGMs) to the mixture of liquid base and crosslinker (10:1 ratio) followed by degassing and heat-assisted crosslinking. We obtained $31 \%$ reduction of thermal conductivity for samples with HGMs content of $20 \%$ by mass. At the same time, sound insulation capacity slightly decreased as a result of lower density of PDMS-HGMs composite in comparison to pure PDMS. The wettability of the samples had no dependence on HGMs content.
\end{abstract}

Keywords: PDMS; Hollow glass microspheres; Thermal conductivity; Sound insulation

\section{Introduction}

Polydimethylsiloxane (PDMS) is the most widely used silicon-based polymer due to its versatility and the whole range of attractive properties that include, but not limited to transparency [1], mechanical flexibility [2], relatively high chemical and thermal stability [3], low toxicity, low solubility in water and high dielectric constant. Nonpolar (hydrophobic) surface of PDMS protects photocatalyst from biofouling [4]. The energy of Si-O bond is $443 \mathrm{~kJ} / \mathrm{mol}$ [5], so PDMS is stable under solar irradiation.

Fabrication of PDMS starts form liquid phase followed by cross-linking to obtain final solid material. This gives opportunity to tune the properties of resulting composite relatively easily by adding other liquids and/or powders into a liquid precursor before cross-linking. One of the apparent properties that can be easily modified by fillers in both directions is thermal conductivity (TC) $[6,7]$. In certain applications of PDMS, enhancement of thermal conductivity is needed, for instance to improve heat dissipation when 
PDMS is used as a sealant in electronics. Carbon-based fillers are considered as the most promising filler candidate [8,9]. Addition of $4.2 \mathrm{wt} \%$ of aligned graphene tubes results in $1082.14 \%$ higher thermal conductivity compared to pure PDMS [7]. One of the most studied enhancer of TC used in PDMS is carbon nanotubes (CNTs) [10-13]. Other fillers reported in literature include carbon black (CB) $[14,15]$, carbon black and CNTs mixture [16], metallic powder [17] and metallic nanowires [18].

Fillers can also increase thermal stability of PDMS at elevated temperatures. Studied filler materials include nanoclay [19], nano-silica [20,21], zirconia or zirconium carbide [22] and others. However, not much works can be found on decreasing TC of PDMS possibly due to already relatively low thermal conductivity of pure PDMS (in the order of $0.2 \mathrm{~W} / \mathrm{K}[23,24]$ ). Nevertheless, further decrease in TC of PDMS can be beneficial in applications where thermal insulation is required like e.g. sealing in construction.

One of the most promising filler materials for thermal insulation in general is silica aerogel [25-29] due to a low density $(0.003-0.1 \mathrm{~g} / \mathrm{cm} 3)$, high porosity $(>99 \%)$, and low thermal conductivity $(0.013-0.04 \mathrm{~W} / \mathrm{m} \cdot \mathrm{K})$. To prevent the absorption of liquid from matrix material during the composite fabrication stage, aerogel should be made either strongly hydrophobic or hydrophilic (depending on matrix composition) e.g. by chemical treatment or additional fabrication steps should be introduced to preserve pores during mixing and solidifying [27-29]. Otherwise, addition of aerogel could even lead to increased thermal conductivity of the final product $[23,30]$. Recently, excellent results in reducing the TC of PDMS-silica aerogel composite were obtained by Lee et al [30] by filling of aerogel pores with ethanol before mixing with PDMS to prevent pores of the aerogel from becoming impregnated with PDMS matrix. After curing at 80C, the ethanol was removed from composite by evaporation and diffusion. Authors succeeded to decrease the heat conductivity of their composite down to $0.018 \mathrm{~W} / \mathrm{m} \cdot \mathrm{K}$.

Despite the high efficiency of silica aerogel fillers, introduction of additional steps makes process more complicated that is not always acceptable for "on-site" fabrication in non-laboratory conditions. Fragile behavior under relatively low external forces is another limiting factor in the use of silica aerogels. Moreover, silica aerogel has a relatively high price due to the need for sophisticated equipment and the long drying process with supercritical fluids [29].

Another candidate as filler for thermally insulating composites is hollow glass microspheres (HGMs) [3133]. Similarly to silica aerogels, HGMs have a low density and excellent thermal insulation properties. However, contrary to silica aerogels, HGMs exhibit a high specific compressive strength and stiffness due to the spherical shape [34]. Moreover, HGMs is more cost-efficient material due to significantly lower manufacture costs compared to aerogels. Encapsulation of gas inside closed rigid spheres prevents them from filling with the matrix material [23]. However, special care still should be taken to avoid breaking of HGMs, since it may result in increase of thermal conductivity up to values exceeding that of a pure matrix material without filler as was shown by Hu et al [35] for silicon rubber (vulcanized product of PDMS) filled with HGMs (diameter 10-100 $\mu \mathrm{m}$ ).

In the present work, we describe fabrication and characterization of composite materials based on PDMS filled with HGMs at various concentration.

\section{Materials and methods}

PDMS matrix was made from Sylgard 184 Silicone Elastomer Kit (Dow Corning, USA), consisting of two liquid components: the base and the curing agent. Hollow glass microspheres (HGMs) (Q-CEL 300, Potters) with tamped bulk density of $0.12 \mathrm{~g} / \mathrm{cm}^{3}$ and particle size ranging from 5 to $200 \mu \mathrm{m}$ (90 $\mu \mathrm{m}$ in average) were used as a heat insulating filler for PDMS matrix. PDMS composites were prepared by adding a predetermined amount of HGMs to PDMS into a freshly prepared liquid mixture of base and curing. Mixing was performed manually with plastic rod to avoid excessive damaging of HGMs by mixing procedure. Base 
to curing agent ratio was kept at 10:1 for all samples as the most common ratio in PDMS applications. Freshly prepared mixture of base, current agent and HGMs was degassed at 300 mbar until no air bubbles remained. Degassed mixture was then poured into the specially prepared vertical cell consisting of two $8 \times 8 \mathrm{~cm}$ glass plates separated by $3.04 \mathrm{~mm}$ thick Teflon spacers and sealed with $5 \mathrm{~mm}$ silicone gasket so that the cell is tightly closed from 3 sides and is opened from above. Glass plates were pressed together and connected by metal clamps. Glass cells filled with PDMS-HGMs mixture were placed into the laboratory furnace for $1 \mathrm{~h}$ at $70^{\circ} \mathrm{C}$. Several samples were prepared with varying concentration of HGMs in PDMS, $0 \%, 2.5 \%, 5 \%, 10 \%$ and $20 \%$ by weight with respect to PMDS.

The distribution of HGMs inside solid PDMS was studied with scanning electron microscope (SEM) (VEGA II, Tescan). Samples for SEM imaging were prepared by tearingsmall pieces of the material from bigger samples to create fresh surfaces for analysis while avoiding damaging the HGM's that would occur in case of cutting by blade.

Density of the samples was determined by measuring the mass and volume of the samples. All samples were $3.04 \mathrm{~mm}$ in thickness and $12.9 \mathrm{~mm}$ in diameter for density measurements.

Thermal conductivity was measured according to ASTM 1114-98 standard by using commercial device THASYS by Hukseflux Thermal Sensors B.V.

Acoustic measurements were performed at $1 \mathrm{kHz}$ sine wave with dynamic microphone Rode M1-S. Sound source was placed in a special soundproof box that has opening $4 \mathrm{~cm}$ in diameter. The opening was covered with one of the samples and reduction in sound level compared to uncovered opening was registered.

Wettability of the samples was tested by drop-casting three $100 \mu \mathrm{L}$ droplets on each sample and measuring the average contact angle.

Finite element simulations of the composite were conducted assuming heat equation in steady state. All materials were modelled directly as geometric primitives: glass microspheres were inserted with their geometrical parameters (diameter, wall thickness) adopted from experimental data. Glass spheres were assumed to be filled with air and were randomly placed into PDMS matrix. Sample was simulated as cube shaped PDMS block with unit edge size. Constant temperature boundary conditions were used at the left and right side of the PDMS block, all other boundaries were considered thermally insulating, replicating, thus modelling symmetry boundary conditions and replicating the block in all directions. Temperature difference between constant temperature boundaries was $10 \operatorname{deg} C$ with $20 \operatorname{deg} C$ at lower limit. As a result of simulation, average heat flux at boundaries was calculated followed by the application of Fourier's law of heat conduction to evaluate the homogenized heat transfer coefficient of the composite. Calculations were conducted using Comsol Multiphysics 5.5.

\section{Results and Discussion}

Addition of HGMs gave the PDMS a white coloration already at $2.5 \%$ concentration due to intense light scattering by the composite, arising from the mismatch of refractive indices of PDMS matrix and HGM's. At $20 \%$ HGM's content $5.8 \mathrm{~mm}$ thick samples became completely opaque (Figure 1). 


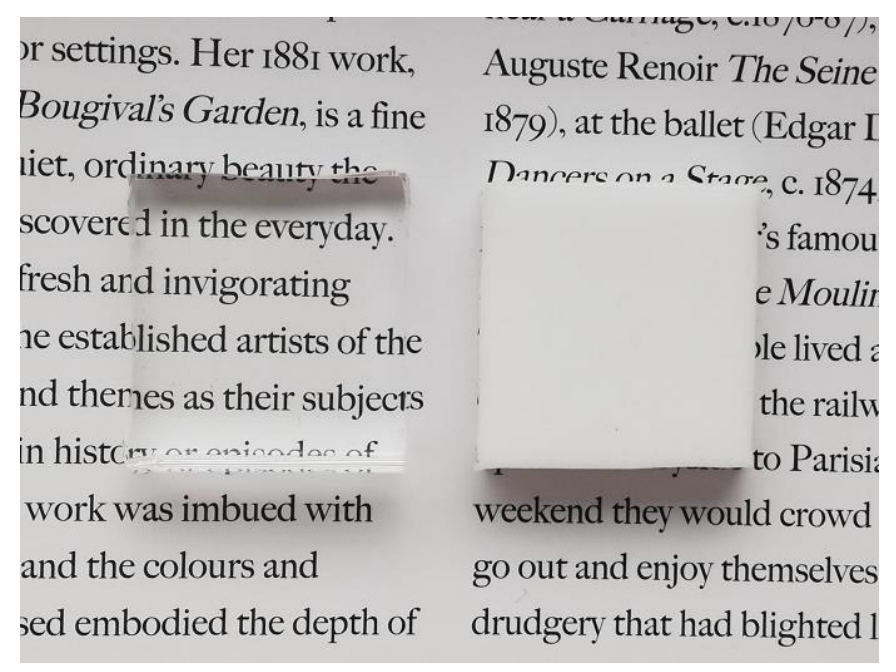

Figure 1. Pure PDMS (left) and PDMS with 20\%Wt HGMs (right). Thickness of both samples is $5.8 \mathrm{~mm}$

Water droplet contact angle measurements did not show any considerable dependence of surface wettability on HGM's content (see Table 1). This is logically explained by the fact that HGMs are entirely embedded into PDMS matrix, so the surface of the composite is basically a pure PDMS.

Table 1. Results of the water droplet contact angle measurements

\begin{tabular}{|c|c|c|c|c|c|}
\hline HGMs content & $0 \%$ & $2.5 \%$ & $5 \%$ & $10 \%$ & $20 \%$ \\
\hline cont. angle 1 & 112 & 116 & 113 & 118 & 114 \\
\hline cont. angle 2 & 116 & 118 & 119 & 118 & 116 \\
\hline cont. angle 3 & 114 & 117 & 116 & 115 & 118 \\
\hline Average angle & $114 \pm 2$ & $117 \pm 1$ & $116 \pm 3$ & $117 \pm 2$ & $116 \pm 2$ \\
\hline
\end{tabular}

SEM observations of torn-off PDMS-HGMs slices revealed that large fraction of HGMs are broken (Figure 2). It is difficult to say if fracturing happened during the mixing and heating stages, or as a result of the sample preparation for SEM imaging. Although the slices with fresh surfaces for SEM analysis were obtained by tearing, not cutting, the process still involves significant mechanical stresses. Most probably, both mixing and tearing were responsible for damage of HGMs. However, contribution of tearing seems to be more significant as closer look reveals that most HGMs that are embedded deeper into the surface of PDMS look undamaged in SEM images (Figure 3). 

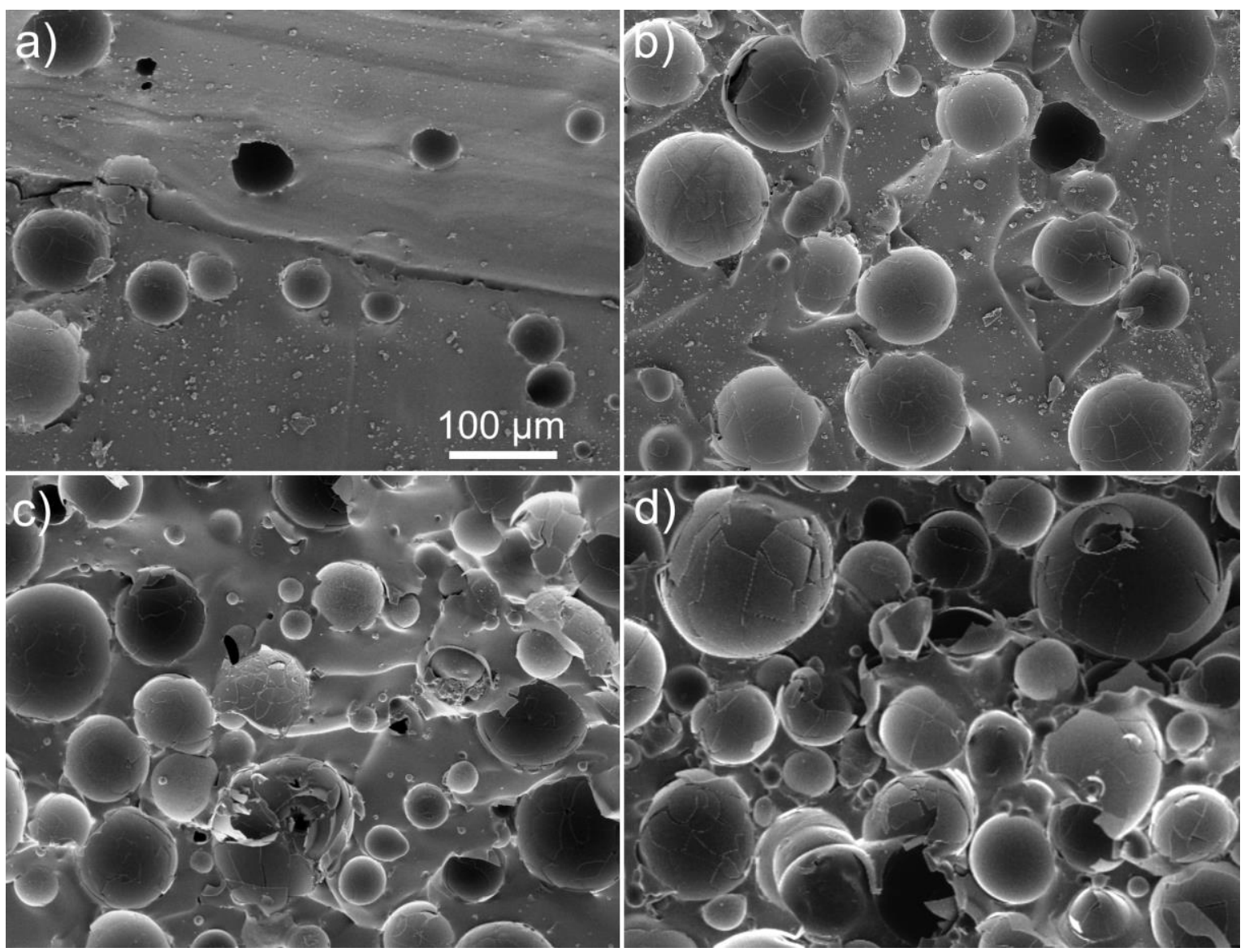

Figure 2. SEM micrographs of PDMS filled with HGMs: $2.5 \%$ (a), $5 \%$ (b), 10\% (c), 20\% (d).

The size of the microspheres also seems to be an important factor in their structural integrity: the majority of sub-100 $\mu \mathrm{m}$ spheres appeared intact under SEM observation (Figure 3).

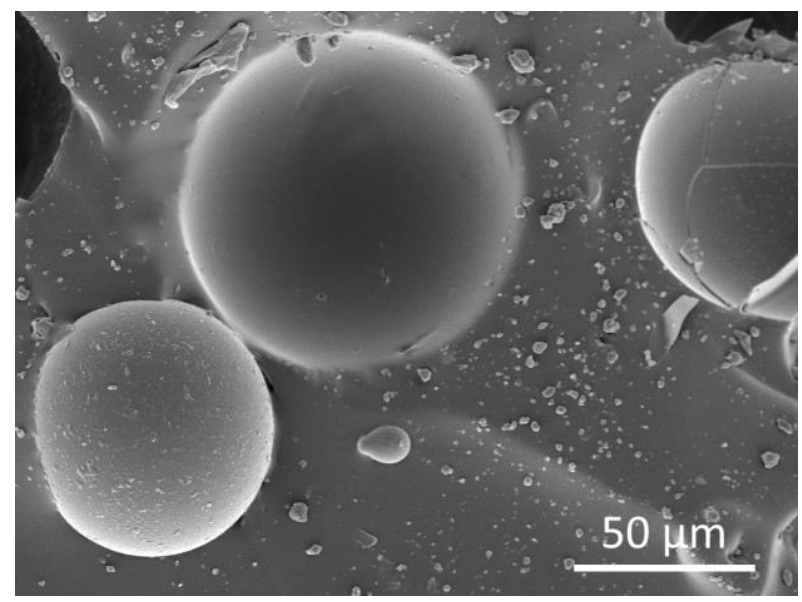

Figure 3. SEM micrograph of HGMs embedded in PDMS. The surface for the observation was obtained by tearing the cured PDMS containing $5 \%$ of HGMs.

The dependence of thermal conductivity and density of PDMS-HGMs composite on HGMs content, alongside with results of FEM simulation, are shown in Figure 4. It can be seen, that both thermal conductivity and density are steadily decreasing with increasing of HGMs content, and are in a good agreement with FEM results. These facts suggest that significant amount of HGMs inside PDMS remained hollow; otherwise, we would observe increase in both thermal conductivity and density 
caused by solid glass shatters that are heavier and better heat conduct than PDMS. In total, we achieved approx. $31 \%$ reduction in thermal conductivity (from 0.161 to $0.111 \mathrm{~W} /(\mathrm{m} \cdot \mathrm{K})$ ) at $20 \% \mathrm{HGMs}$ content. This result is far from the one obtained by Lee et al [30] for specially treated aerogel filler. However, the use of HGMs benefits from simplicity of fabrication process as it does not require additional steps for preserving hollow structure and therefore can be performed outside of the laboratory conditions without the use of special equipment. In contrast, addition of silica aerogel without the pore restoration procedure results in increased thermal conductivity of PDMS and other organic materials $[23,30]$. Therefore, the use of HGMs instead of silica aerogel particles can be justified in certain practical applications such as on-site preparation of sealer with enhanced thermal insulation. It should be noted, that addition of HGMs above $20 \%$ is problematic due to high viscosity of the solution making it difficult to pour.

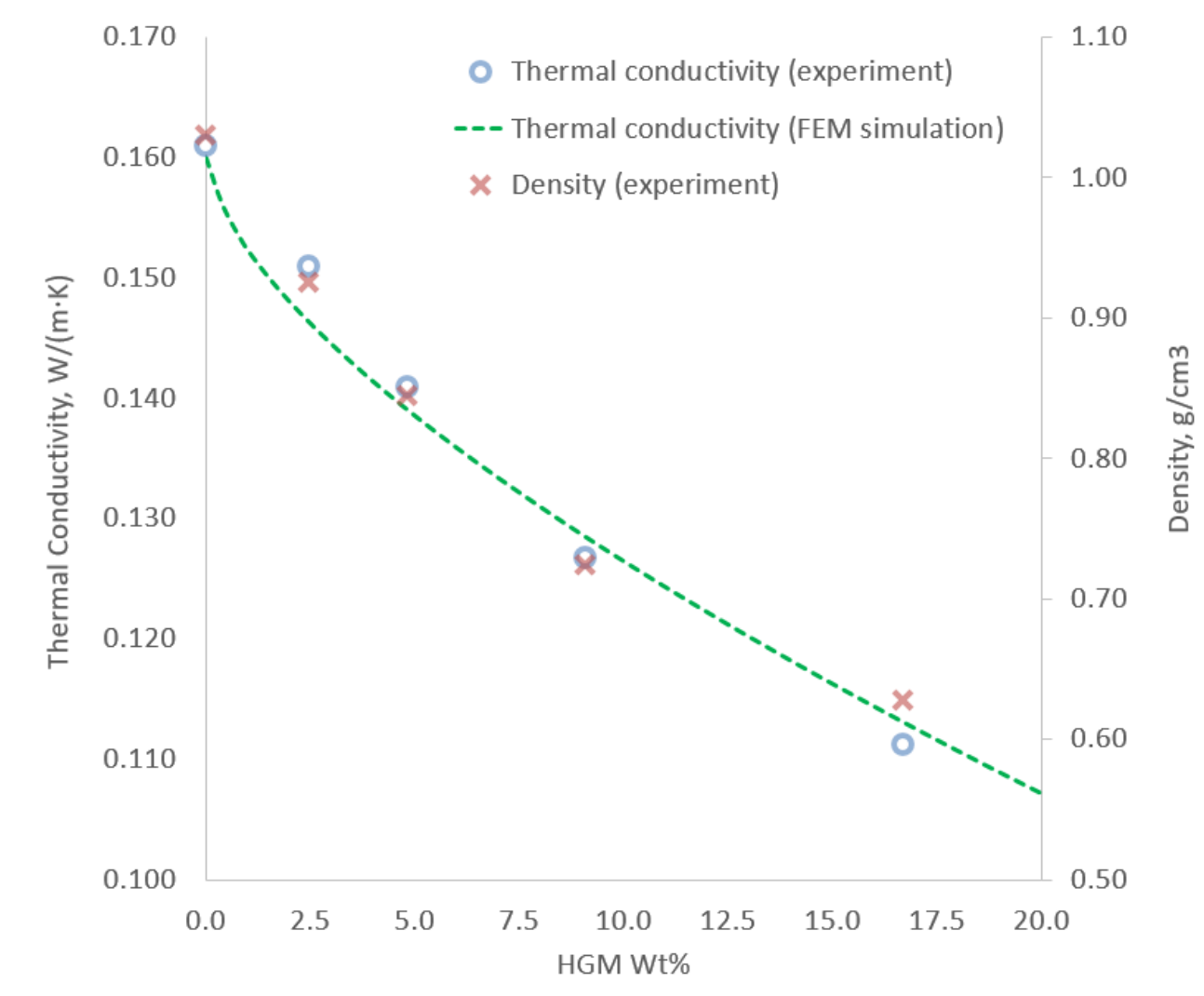

Figure 4. The dependence of thermal conductivity and density of PDMS-HGMs composite on HGMs content

Alongside with heat insulation, sound insulation is another property that can be desirable in sealing applications. Our measurements have shown that addition of HGMs resulted in slightly decreased sound insulation capacity from $-29 \mathrm{~dB}$ for pure PDMS to $-26 \mathrm{~dB}$ for PDMS with $20 \% \mathrm{HGMs}$ as tested on $5.08 \mathrm{~mm}$ thick samples for $1 \mathrm{kHz}$ sound frequency (commonly used frequency in acoustic tests as it corresponds well to the frequency of human voice). The ability to absorb sound energy is related both to structure and to density (mass) of the object. In our case, higher density of pure PDMS was determining factor for more efficient sound insulation.

\section{Conclusions}

A novel composite material with low thermal conductivity have been prepared by adding hollow glass microspheres (HGMs) to the mixture of liquid base and cross-linker (10:1 ratio) of PDMS, followed by degassing and heat-assisted crosslinking for $1 \mathrm{~h}$ at $70^{\circ} \mathrm{C}$. HGMs concentration in the samples was varied 
from $0 \%$ to $20 \%$ by weight. Addition of HGM's gave PDMS an opaque white appearance. Water droplet contact angle measurements did not show any considerable dependence of surface wettability on HGMs content. This is logically explained by the fact that HGMs are entirely embedded into PDMS matrix so the surface of the composite is basically a pure PDMS. Thermal conductivity and density steadily decreased with increase of HGM's concentration, reaching $31 \%$ reduction in thermal conductivity (from 0.161 to $0.111 \mathrm{~W} /(\mathrm{m} \cdot \mathrm{K})$ ) and $39 \%$ reduction in density (form 1.03 to $0.63 \mathrm{~g} / \mathrm{cm}^{3}$ ) at $20 \% \mathrm{HGM}$ 's concentration in comparison to pure PDMS. At the same time sound insulation capacity of $5.08 \mathrm{~mm}$ thick samples decreased from $-29 \mathrm{~dB}$ for pure PDMS to $-26 \mathrm{~dB}$ for PDMS with $20 \% \mathrm{HGMs}$ content as measured at $1 \mathrm{kHz}$ sound frequency. This is a price paid for lower density of PDMS-HGM's composite in comparison to pure PDMS. Thermal conductivity values obtained in this work were higher as compared to those obtained by Lee et al [30] for specially treated aerogel filler. However, the use of HGM's in preparation of composites with PDMS benefits from simplicity of fabrication as it does not require additional steps for preserving hollow structure and therefore can be performed outside of the laboratory conditions without the use of special equipment. Therefore, the use of HGMs instead of silica aerogel particles can be justified in certain practical applications such as on-site preparation of sealer with enhanced thermal insulation.

\section{Acknowledgements}

This work was supported by Russian Science Foundation project grant 18-19-00645 "Adhesion of polymerbased soft materials: from liquid to solid" and by the Estonian Centre of Excellence in Zero Energy and Resource Efficient Smart Buildings and Districts, ZEBE, grant 2014-2020.4.01.15-0016 funded by the European Regional Development Fund, ERA Chair MATTER from the European Union's Horizon 2020 research and innovation programme under grant agreement No 856705.

\section{References}

[1] Cambié D, Bottecchia C, Straathof N J W, Hessel V and Noël T 2016 Applications of Continuous-Flow Photochemistry in Organic Synthesis, Material Science, and Water Treatment Chem. Rev. 116 10276-341

[2] Lamberti A 2015 Microfluidic photocatalytic device exploiting PDMS/TiO2 nanocomposite Appl. Surf. Sci. 335 50-4

[3] Alder K I and Sherrington D C 1998 Synthesis of spherical particulate polysiloxane resins as catalyst supports Chem. Commun. 131-2

[4] Wooh S, Encinas N, Vollmer D and Butt H-J 2017 Stable Hydrophobic Metal-Oxide Photocatalysts via Grafting Polydimethylsiloxane Brush Adv. Mater. 291604637

[5] Zhu Y F, Pan K L, Jiang L and Dan Y 2007 Preparation and evaluation of photocatalytic activity of poly(dimethylsiloxane)-titanium dioxide composites Plast. Rubber Compos. 36 360-4

[6] He X, Huang Y, Wan C, Zheng X, Kormakov S, Gao X, Sun J, Zheng X and Wu D 2019 Enhancing thermal conductivity of polydimethylsiloxane composites through spatially confined network of hybrid fillers Compos. Sci. Technol. 172 163-71

[7] Zhang Y-F, Ren Y-J, Guo H-C and Bai S 2019 Enhanced thermal properties of PDMS composites containing vertically aligned graphene tubes Appl. Therm. Eng. $150840-8$

[8] Ngo I-L, Jeon S and Byon C 2016 Thermal conductivity of transparent and flexible polymers containing fillers: A literature review Int. J. Heat Mass Transf. 98 219-26 
[9] Chowdhury S, Olima M, Liu Y, Saha M, Bergman J and Robison T 2016 Poly dimethylsiloxane/carbon nanofiber nanocomposites: fabrication and characterization of electrical and thermal properties Int. J. Smart Nano Mater. 7 236-47

[10] Ha J-U, Hong J, Kim M, Choi J K, Park D W and Shim S E 2013 Improvement of Thermal Conductivity of Poly(dimethyl siloxane) Composites Filled with Boron Nitride and Carbon Nanotubes Polym.

Korea 37 722-9

[11] Hong J, Lee J, Hong C K and Shim S E 2010 Improvement of thermal conductivity of poly(dimethyl siloxane) using silica-coated multi-walled carbon nanotube J. Therm. Anal. Calorim. 101 297-302

[12] Hong J, Lee J, Hong C K and Shim S E 2010 Effect of dispersion state of carbon nanotube on the thermal conductivity of poly(dimethyl siloxane) composites Curr. Appl. Phys. 10 359-63

[13] Hong J, Lee J, Jung D and Shim S E 2011 Thermal and electrical conduction behavior of alumina and multiwalled carbon nanotube incorporated poly(dimethyl siloxane) Thermochim. Acta 512 34-9

[14] Chen H, Botef I, Guduri B, Srinivasu V V, Giri P K, Goswami D K, Perumal A and Chattopadhyay A 2010 Thermal and Bonding Properties of Nano Size Carbon Black Filled PDMS INTERNATIONAL CONFERENCE ON ADVANCED NANOMATERIALS AND NANOTECHNOLOGY (ICANN-2009) (Guwahati, Assam (India)) pp 243-8

[15] Chen H, Botef I, Zheng H, Maaza M, Rao V V and Srinivasu V V 2011 Thermal conductivity and stability of nanosize carbon-black-filled PDMS: fuel cell perspective Int. J. Nanotechnol. 8437

[16] Hong J, Park D W and Shim S E 2012 Electrical, thermal, and rheological properties of carbon black and carbon nanotube dual filler-incorporated poly(dimethylsiloxane) nanocomposites Macromol. Res. 20 465-72

[17] Chuang H-S and Wereley S 2009 Design, fabrication and characterization of a conducting PDMS for microheaters and temperature sensors J. Micromechanics Microengineering 19045010

[18] Balachander N, Seshadri I, Mehta R J, Schadler L S, Borca-Tasciuc T, Keblinski P and Ramanath G 2013 Nanowire-filled polymer composites with ultrahigh thermal conductivity Appl. Phys. Lett. 102 093117

[19] Smitha Alex A, Rajeev R S, Krishnaraj K, Sreenivas N, Manu S K, Gouri C and Sekkar V 2017 Thermal protection characteristics of polydimethylsiloxane-organoclay nanocomposite Polym. Degrad. Stab. 144 281-91

[20] Chen D, Chen F, Hu X, Zhang H, Yin X and Zhou Y 2015 Thermal stability, mechanical and optical properties of novel addition cured PDMS composites with nano-silica sol and MQ silicone resin Compos. Sci. Technol. 117 307-14

[21] Gu W J, He B G and Li Y 2012 A New Kind of Silica Microspheres Used as Filler for PDMS Composite Adv. Mater. Res. 534 184-7

[22] Yang D, Zhang W, Jiang B and Guo Y 2013 Silicone rubber ablative composites improved with zirconium carbide or zirconia Compos. Part Appl. Sci. Manuf. 44 70-7

[23] Vahtrus M, Oras S, Antsov M, Reedo V, Mäeorg U, Lõhmus A, Saal K and Lõhmus R 2017 Mechanical and thermal properties of epoxy composite thermal insulators filled with silica aerogel and hollow glass microspheres Proc. Est. Acad. Sci. 66339 
[24] Al-Khudary N, Cresson P Y, Orlic Y, Coquet P, Pernod P and Lasri T 2014 Measurement of the Thermal Conductivity of Polydimethylsiloxane Polymer Using the Three Omega Method Key Eng. Mater. 613 259-66

[25] Ge D, Yang L, Li Y and Zhao J 2009 Hydrophobic and thermal insulation properties of silica aerogel/epoxy composite J. Non-Cryst. Solids 355 2610-5

[26] Kim G S and Hyun S H 2003 Effect of mixing on thermal and mechanical properties of aerogel-PVB composites J. Mater. Sci. 38 1961-6

[27] Kim S Y, Noh Y J, Lim J and You N-H 2014 Silica aerogel/polyimide composites with preserved aerogel pores using multi-step curing Macromol. Res. 22 108-11

[28] Kim H M, Noh Y J, Yu J, Kim S Y and Youn J R 2015 Silica aerogel/polyvinyl alcohol (PVA) insulation composites with preserved aerogel pores using interfaces between the superhydrophobic aerogel and hydrophilic PVA solution Compos. Part Appl. Sci. Manuf. 75 39-45

[29] Kim H M, Kim H S, Kim S Y and Youn J R 2015 Silica aerogel/epoxy composites with preserved aerogel pores and low thermal conductivity E-Polym. 15 111-7

[30] Lee H, Lee D, Cho J, Kim Y-O, Lim S, Youn S, Jung Y C, Kim S Y and Seong D G 2019 Super-insulating, flame-retardant, and flexible poly(dimethylsiloxane) composites based on silica aerogel Compos. Part Appl. Sci. Manuf. 123 108-13

[31] Zhu B, Ma J, Wang J, Wu J and Peng D 2012 Thermal, dielectric and compressive properties of hollow glass microsphere filled epoxy-matrix composites J. Reinf. Plast. Compos. 31 1311-26

[32] Liang J Z and Li F H 2006 Measurement of thermal conductivity of hollow glass-bead-filled polypropylene composites Polym. Test. 25 527-31

[33] Yung K C, Zhu B L, Yue T M and Xie C S 2009 Preparation and properties of hollow glass microsphere-filled epoxy-matrix composites Compos. Sci. Technol. 69 260-4

[34] Trofimov A, Pleshkov L and Back H 2006 Hollow glass microspheres for high strength composite cores Reinf. Plast. 50 44-50

[35] Hu Y, Mei R, An Z and Zhang J 2013 Silicon rubber/hollow glass microsphere composites: Influence of broken hollow glass microsphere on mechanical and thermal insulation property Compos. Sci. Technol. 79 64-9 